\title{
Prever y preveer: historia de dos palabras
}

\author{
Carlos González Vergara* \\ Trinidad Cabezón Droguett \\ Francisca Toro Varela \\ Pontificia Universidad Católica de Chile
}

\begin{abstract}
Resumen
En oposición a la frecuente explicación de que la voz preveer se origina en un cruce erróneo entre prever y proveer, la presente investigación plantea la hipótesis de que preveer es una variante que ha existido a lo largo de la historia del español en competencia con prever, y que solo comenzó a ser proscrita en el español culto luego de la aparición de Diccionario de autoridades (1726-1739). Los autores sustentan esta hipótesis en dos hechos principales: (a) todas las obras lexicográficas españolas anteriores al año 1726 muestran el vocablo preveer y no prever, el que aparece por primera vez en el primer diccionario de la RAE y es replicado luego en las obras lexicográficas posteriores; y (b) el análisis del registro histórico del español, realizado sobre el Corpus diacrónico del español (CORDE), análisis que muestra apariciones escritas tanto de la forma prever como preveer hasta mediados del siglo XVIII, momento en el cual prever pasa a ser la variante privilegiada y que coincide con la aparición del Diccionario de autoridades.
\end{abstract}

Palabras clave: preveer, prever, historia del español, léxico, Diccionario de autoridades.

Para correspondencia, dirigirse a: Carlos González Vergara (cgonzalv@uc.cl), Departamento de Ciencias del Lenguaje, Facultad de Letras - Pontificia Universidad Católica de Chile - Av. Vicuña Mackenna 4860, Macul, Santiago, Chile. 


\title{
PREVER \& PREVEER: A TALE OF TWO WORDS
}

\begin{abstract}
In contrast to the frequent claim that the Spanish verb preveer ('foresee') is originated in the mistaken combination of prever and proveer ('provide'), this research holds the hypothesis that preveer is a variant that has existed along the history of Spanish, competing with prever. It also claims that preveer only started to be proscribed in formal Spanish after the publication of the Diccionario de autoridades (1726-1739). The authors substantiate this hypothesis with two main facts: (a) all Spanish dictionaries prior to 1726 show the word preveer and not prever, which just appears in the first dictionary published by the Real Academia Española, and it is reproduced in later lexicographic works; (b) the analysis of the historical registers of Spanish carried out in the Corpus diacrónico del español (CORDE) shows written occurrences of both forms, prever and preveer. Prever does not become the privileged variant until the mid-18th century, date that coincides with the publication of the Diccionario de autoridades.
\end{abstract}

Keywords: History of Spanish, Lexicography, Preveer, Prever (foresee), Diccionario de autoridades.

Recibido: 14/09/16 Aceptado: 10/07/17

\section{INTRODUCCIÓN ${ }^{1}$}

El verbo preveer tiene mala fama. El blog El español sin misterios (Correa 2011), por ejemplo, indica que es una voz que no existe en español, aunque-según testimonia paradójicamente- "goza de mucha popularidad". Para Seco (1961) se trata de un "vulgarismo", mientras que la Fundación del Español Urgente (2011) lo cataloga de "incorrección", al igual que hace el Diccionario panhispánico de dudas (RAE 2005). El Centro Virtual Cervantes

1 Este artículo corresponde a una versión corregida y ampliada de la ponencia presentada en I Coloquio de Lingüistica Histórica, organizado en la Pontificia Universidad Católica de Chile en octubre de 2015. Los autores agradecen los valiosos comentarios recibidos en esa ocasión. 
(s. f.), por su parte, lo incluye dentro de su sección Museo de los horrores ${ }^{2}$. Asimismo, como puede apreciarse en la siguiente cita, la Academia Chilena

2 Las siguientes son las citas completas de las fuentes mencionadas:

El verbo "preveer" no existe en español. Sin embargo, goza de mucha popularidad entre algunas personas. Así, no es inusual toparse con oraciones de este estilo: "tienes que preveer las dificultades que se van a presentar"; "Juan prevee gastar poco dinero en la primera fase del proyecto"; "ellos no preveyeron que el computador pudiera fallar".

Todas estas oraciones son incorrectas. "Prever", con una sola $e$, es el verbo indicado en los ejemplos anteriores [...] Este error proviene del cruce de dos verbos españoles: "prever" y "proveer". Como la pronunciación de estos verbos tiende a confundirse, es bastante probable que esta confusión haya generado la forma híbrida "preveer" que compite, en el uso, con la forma correcta "prever". (El español sin misterios 2011)

prever. Verbo irregular. Se conjuga como ver [34]. No debe confundirse en su conjugación con proveer, ni en su significado con prevenir (-» PREVENIR, 3). Es vulgarismo frecuente decir preveer y atribuir a este verbo la conjugación 17 (como leer): «Hay que preveer, planear y ejecutar» (L. Pedret, Vanguardia, 21.1.1962, 21); "[Películas] realizadas directamente para la televisión, aunque preveyendo su difusión en las salas» (Informaciones, Supl., 26.1.1978, 11). (Seco 1961)

El verbo prever está formado por el verbo ver y el prefijo pre, es decir 'ver antes', y debe conjugarse igual que ver. A veces, se mezclan los verbos prever y proveer, lo que da lugar a la creación del verbo preveer. De ahí que resulten formas como preveyó, preveyera o preveyendo, en lugar de previó, previera o previendo. Son incorrectas, por tanto, frases como «Preveyó graves problemas para los trabajadores de las petroleras», «Los directivos de la empresa no preveyeron los problemas económicos» o «Un contestador automático pedía que no bloquearan la línea, quizás preveyendo que los familiares de los afectados...». Este error se extiende a todos los tiempos y formas del verbo prever: prevee, preveemos, preveímos, etc. en lugar de prevé, prevemos, previmos, etc. (Fundéu 2011)

prever. 'Ver [algo] con anticipación', 'conjeturar [algo futuro]' y 'disponer [lo necesario] para futuras contingencias'. Verbo irregular: se conjuga como ver $(\rightarrow$

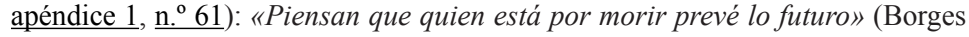
Libro [Arg. 1975]). Es incorrecta la grafía * preveer, debida al cruce con proveer ('suministrar'; $\rightarrow$ proveer(se)), cuyo modelo es leer; por tanto, son incorrectas formas como *prevee, *preveyó, *preveyendo, etc., en lugar de prevé, previó, previendo, etc.: * «Un hombre que prevee su muerte con 10 años de anticipación y no cumple, no es serio» (Nacional [Ven.] 12.1.97). (RAE 2005)

Hemos recibido el siguiente mensaje: "Un verbo que está habitualmente mal conjugado es el verbo prever. Incluso en infinitivo he visto muchas veces preveer. $\mathrm{Y}$ al conjugarlo he visto tú prevees, nosotros preveemos, cuando la conjugación de este verbo es idéntica a la del verbo ver.". Efectivamente, es bastante común el uso de * preveer en lugar de prever. Debido a su parecido con proveer se cruzan las conjugaciones de los verbos prever y proveer. (Centro Virtual Cervantes, s.f.) 
de la Lengua $(2007,2014)$ también estima inaceptable su uso: "El infinitivo * preveer (resultado del cruce de prever con proveer) no es aceptable, como tampoco cualquiera de las formas conjugadas de este verbo (* prevee por prevé, * preveyó por previó, *preveyendo por previendo, etc.)”. Finalmente, tanto la Real Academia Española (RAE) como la Asociación de Academias de la Lengua Española (ASALE) siguen también esta tendencia en la Nueva gramática de la lengua española, como se observa en el párrafo dedicado a este tema (2009: $242 \S 4.12 \mathrm{i})$ :

A pesar de que ver no ha seguido el mismo modelo de leer o creer y ha reducido a una sola las dos vocales -e- en secuencia tautosilábica (lee, cree, pero ve no *vee), se piensa que la sílaba perdida todavía ejerce cierta influencia en el paradigma al que pertenece. A ese influjo cabe atribuir formas anómalas como preveemos o preveyeron, atestiguadas en la lengua oral de muchos países, en lugar de las variantes recomendadas prevemos, previeron, aunque también es posible que la existencia de estas formas se deba al cruce con proveer.

Todos estos textos coinciden también en señalar que la palabra correcta o recomendada es prever, formada por la adición del prefijo pre- al verbo ver. Esta formación parece ser clara y transparente. La explicación que estas fuentes y otras parecidas suelen dar para la formación incorrecta es que se trata de un cruce con el verbo proveer.

Tres aspectos llaman la atención de estas descripciones.

(1) La alta prevalencia de la forma "incorrecta" preveer entre los hablantes. Todos los textos consultados coinciden en afirmar que preveer es una palabra muy común, incluso entre hablantes de alta cultura. Véase al respecto esta cita del texto La perversión del lenguaje (de Miguel 1985: 66): "Tengo visto muchas veces en los exámenes de los alumnos el curioso verbo preveer, que nunca ha existido en castellano. Es posible que exista, pues esos alumnos se licencian, y aun se doctoran, y llegan algunos a puestos de cierta preeminencia, y siguen conjugando ese divertido verbo. Hacen declaraciones y se someten a entrevistas. Llegan a altos cargos y siguen diciendo preveer, e incluso preveyó, cada vez con mayor aplomo. Estoy resignado."

(2) La formación lexicogenésica propuesta para el verbo catalogado de correcto ( prever $<$ pre- ver). Esta historia derivativa es composicionalmente transparente, condición que debería influir en los hablantes para que usaran esta forma y no la alternativa incorrecta preveer, que -como queda dicho- es, sin embargo, muy frecuente. A esto se debe añadir que, si bien es sincrónicamente plausible, esta explicación es históricamente falsa, dado que tanto prever como proveer derivan del mismo verbo latino videre: prever 
$<$ prae- videre y proveer < pro- videre. Así lo testimonian Corominas (19541957) y Corominas y Pascual (1991-1997), quienes señalan que prever es una adaptación del latín praevidere, con idéntico significado ${ }^{3}$. Cabe, entonces, preguntarse por qué estos dos verbos evolucionaron de manera distinta (uno simplificado en ver y el otro manteniendo la duplicación de la vocal e) o, cuestión más interesante para nuestros propósitos, si esta evolución diferenciada realmente ocurrió.

(3) La explicación propuesta de que la forma incorrecta preveer proviene de un cruce moderno con proveer, verbo con el que no se relaciona léxicamente de manera transparente en el español actual.

Nuestra investigación presenta una propuesta alternativa a la hipótesis comentada en el punto (3). Proponemos que preveer no proviene de un cruce entre los verbos prever y proveer, sino que preveer es una forma que ha existido a lo largo de la historia del español en competencia con prever, y que incluso fueron formas igualmente populares hasta mediados del siglo XVIII, momento en el que, con la aparición del Diccionario de autoridades (RAE 1726-1739), la forma prever fue elegida por sobre preveer, la que quedó proscrita en el español culto.

Para probar esta hipótesis nos apoyaremos en dos argumentos: (1) la consulta sobre el tratamiento que ambas voces han recibido históricamente en obras lexicográficas del español contenidas en el Nuevo tesoro lexicográfico de la lengua española y en el sitio Memoria chilena, y (2) la pesquisa del uso registrado de las dos formas entre los siglos x y XIX en el Corpus diacrónico del español.

La estructura de este artículo es la siguiente. En la sección 2 presentamos las investigaciones lingüísticas emprendidas en relación con este tema hasta el momento. En la sección 3 revisamos las obras lexicográficas históricas que recogen el uso y las recomendaciones normativas sobre los verbos prever y preveer, tanto las contenidas en el Nuevo tesoro lexicográfico de la lengua española, como en las obras pertinentes recogidas en Memoria chilena. En la sección 4 presentamos los resultados de un estudio basado en datos del Corpus diacrónico del español. Finalmente, en la sección 5 exponemos nuestras conclusiones.

\footnotetext{
Es interesante notar que estos autores indican en sus obras que la forma más antigua de la que tienen registro es preveer y que, de manera concordante con nuestra propuesta, prever no aparece sino hasta el Diccionario de autoridades.
} 


\section{ESTADO DE LA CUESTIÓN}

Curiosamente, y en contraste con la clara atención que a este vocablo prestan los textos normativos, apenas existen estudios propiamente lingüísticos que traten del verbo preveer. En este apartado presentamos los dos más pertinentes: la explicación basada en el fenómeno del archisilabismo, de Alcoba Rueda (2012) y la propuesta histórica de Rini (1991 y 2001).

\subsection{PreVEer Como un CASO De ARChisilabismo}

En el estudio que realiza Alcoba Rueda (2012), en el que sistematizó la información en torno a los casos existentes de palabras archisílabas y en el que definió las propiedades y características de estas, se nombra el caso de prever y preveer, y se postula la existencia de este último vocablo como un ejemplo de creación archisílaba. Según el autor, este término fue propuesto por Arteta (1995) en su artículo "La moda del archisílabo", en que lo define como palabras que sustituyen a otros conceptos de igual o similar significado y que son preferidas por los hablantes por ser más extensas que el término desplazado. Se consideran, así, como "neologismos que podemos situar en el cuadrante de la excepción, de lo marcado, de lo raro y de lo asistémico, porque son creaciones para conceptos aparentemente ya satisfechos en las palabras postergadas" (p. 20). Ejemplos de archisilabismo serían los usos de diferenciación frente a diferencia, problemática frente a problema o visualizar frente a ver.

Según el análisis de Alcoba Rueda -quien se basa en los datos entregados por De Miguel (1985), Arteta $(1995,2005,2008,2010)$ y otros recogidos por el mismo autor- la palabra archisílaba se caracterizaría por poseer más afijos y sílabas que la palabra postergada, lo que se traduciría en un mayor peso léxico, y le otorgaría al archisílabo su carácter de "rareza". Además, según el autor, la palabra archisílaba es típicamente monosémica frente a la polisemia de la palabra a la que sustituye; es decir, su uso tiende a ser más especializado en comparación con el sentido de la palabra postergada. En esta línea, si bien en el estudio no se trata con detalle el caso de preveer, se puede inferir que se plantea esta palabra como archisílaba dado que tiene una sílaba más que su postergada prever. Alcoba Rueda (2012: 21) señala que "[s]i nos fijamos en el término y algunas ideas apuntadas por Arteta, los datos tienen que ver con las sílabas [...], con el significado [...], y con alguna otra razón, además del número de sílabas, que explique el uso de 'ética' en vez de moral o de 'preveer' en vez de prever". 
Se puede apreciar, sin embargo, que a pesar de que preveer podría poseer las condiciones fonológicas propias de un archisílabo, esta palabra no cumple con la relación semántica que debería establecerse entre el archisílabo y su palabra postergada: preveer no acota el significado de prever, ni tampoco le da un sentido más especializado, sino que ambas formas verbales aluden al mismo evento, sin distinción alguna. Nos parece, en consecuencia, que la inclusión de preveer como una creación fundada en el archisilabismo es una explicación poco satisfactoria.

\subsection{LA PROPUESTA HISTÓRICA DE RINI}

Otra investigación que recoge este tema, con seguridad la más relevante, es el estudio de Rini (1991), quien trabaja la evolución histórica de /-ee-/ $>$ /-e-/ en verbos que en el español antiguo presentan la vocal duplicada, como veer, lexema base de prever. Este autor afirma que en el tránsito del español antiguo al español moderno la tendencia regular fue simplificar las vocales dobles a vocales simples. Así, veer sufrió una contracción a ver al igual que seer a ser, a diferencia de lo que ocurrió con verbos como proveer, que, a pesar de tener como lexema base veer, mantuvieron la vocal doble (Rini 2001).

En vista de esto, con el objetivo de explicar la falta de reducción vocálica en verbos con /-ee-/ original (como proveer, leer, creer y poseer), el autor revisa las distintas conjugaciones de sus bases léxicas. En este análisis detecta en el pretérito imperfecto del indicativo de prever la forma antigua de ver: veía, veías, etc., formas consideradas actualmente irregulares (dado que retienen una vocal en vez de perderla, como era la tendencia normal), pero perfectamente regulares si se considera que están basadas en el antiguo infinitivo del español: veer < videre. Lathrop (1980: 183) explica: "El imperfecto irregular español veía es el reflejo del resultado regular del imperfecto de veer en español antiguo".

El pretérito imperfecto es, en efecto, uno de los ejes centrales en los trabajos de Rini (2001), pues lo utiliza como argumento desde su teoría de la difusión léxica para afirmar que la reducción de /-ee-/ > /-e-/ no fue sistemática y que el actual pretérito imperfecto del español (veía) es la evidencia de la productividad del imperfecto de veer y no de su forma moderna ver. Este punto de la investigación de Rini es particularmente importante para efectos de nuestra investigación, pues a partir de él podemos establecer como cierta la existencia histórica de preveer, dado que actualmente la forma prevalente del pretérito imperfecto de prever es 
preveía (forma correspondiente a preveer) y no *prevía (del paradigma de prever), como debió ser por cambio fónico regular.

El segundo eje fundamental de la investigación de Rini es el vínculo que establece entre infinitivos y participios pasados de estos verbos, relacionados por el uso de auxiliares. Así, señala Rini (1991: 98), mientras el infinitivo se sirve de verbos conjugados (por ejemplo, debo en debo comer), el participio emplea el auxiliar haber (por ejemplo, he comido). Esta relación entre ambos haría que la estructura del participio influyera en la cantidad silábica del infinitivo. Rini señala que existe un patrón en estos verbos no contractos: el participio pasado tiene una sílaba más que su infinitivo. Así, creer tiene dos sílabas, mientras que creído tiene tres. Lo mismo ocurre con leer/leído y poseer/poseído.

Según el autor, la tendencia de aumentar en una sílaba el participio se habría vuelto tan regular que, incluso los infinitivos que aún no sufrían la contracción se habrían "reacomodado" para no interferir con este patrón. En sus palabras (p. 99): "it appears the syllabic rhythm of the past participle has influenced the infinitive to retain an extra syllable, impeding the contraction of /-ee-/ > /-e-/"'. No obstante, según el autor, en el caso de veer el cambio habría ocurrido a la inversa: para no interferir con dicho patrón fue necesario hacer la contracción: ver posee una sílaba, mientras que visto posee dos. Lo mismo habría ocurrido también con ser/sido.

A nuestro juicio, sin embargo, esta explicación se presenta como poco consistente, pues, por un lado, propone un vínculo entre infinitivos y participios que no se justifica de manera clara; y, por otro, no aborda la forma irregular (regular en su tiempo) del participio de prever: preveído, sino que solo considera la forma moderna, previsto, para aclarar el fenómeno, mientras hace lo contrario al explicar la formación de proveer, descrita desde su forma proveído y no como provisto.

\section{OBRAS LEXICOGRÁFICAS}

Revisamos a continuación un conjunto de obras lexicográficas históricamente relevantes que contienen alguna de las formas prever o preveer. Este análisis es, a nuestro juicio, pertinente, pues la inclusión de uno u otro vocablo y sus tratamientos nos entregan pistas sobre la difusión y apreciación de la forma estigmatizada preveer. 


\subsection{Obras del NueVo teSORO LEXICOGRÁFICO DE LA LENGUA ESPAÑOLA}

El Nuevo tesoro lexicográfico de la lengua española (en adelante NTLLE) es un repertorio bibliográfico de consulta abierta reunido por la Real Academia Española. Se trata, en palabras de la RAE, de "un diccionario que contiene todo el léxico de la lengua española desde el siglo xv hasta el xx, tal y como es recogido, sistematizado, definido e inventariado por los más importantes repertorios lexicográficos, sean monolingües o bilingües, dedicados a la lengua española"4.

En esta base bibliográfica hicimos el rastreo de las apariciones de las formas prever y preveer, y su tratamiento. La primera cuestión que llama la atención es que el vocablo preveer registra apariciones más antiguas que prever. La primera inclusión de preveer ocurre en una obra de 1604, mientras que prever no se registra sino hasta 1726 como una palabra propia del español. Debe notarse que, de las ocho obras que incluyen preveer entre 1604 y 1721, ninguna de ellas juzga esta palabra como una incorrección o un vicio idiomático, sino que -por el contrario- no se le otorga marca alguna, que ninguno de estos ocho textos más tempranos registra la variante prever $\mathrm{y}$, finalmente, que estas obras son diccionarios bilingües (creados para el conocimiento del español para hablantes de otras lenguas), todo lo cual muestra que sus autores consideraron la forma preveer como la variante más común de esta palabra en el castellano.

La primera obra que incluye preveer es el Diccionario muy copioso de la lengua española y francesa, de Ioan Palet, editado en París en $1604^{5}$. La entrada pertinente se presenta en $(1)^{6}$, y en ella puede observarse tanto la falta de marcas de incorrección como la notoria ausencia de la palabra prever.

4 Fuente: http://www.rae.es/recursos/diccionarios/diccionarios-anteriores-1726-1992/ nuevo-tesoro-lexicografico

5 Existen dos obras lexicográficas que preceden al diccionario de Palet, pero que no aparecen recogidos en el NTLLE. Se trata del Recuil de dictionaries Francois, Espagnols et Latins, de Heinrich Hornkens (Bruselas, 1599), y el A dictionary in Spanish and English, de John Minsheu (Londres, 1599). En ambos se incluye la variante preveer y no prever, tal como puede consultarse en Nieto y Alvar (2007). Los autores agradecen al Dr. Darío Rojas por esta información.

${ }^{6}$ En todas las transcripciones se ha respetado la ortografía y peculiaridades tipográficas de los originales. 
(1) preueer, Preuoír (Palet 1604)

Preveer (sin marcas y con ausencia de prever) aparece luego en el Tesoro de las dos lenguas francesa y española, de César Oudin (París 1607):

(2) preueer, Preuoir. (Oudin 1607)

El siguiente diccionario pertinente es el Tesoro de las tres lengvas francesa, italiana y española, de Girolamo Vittori (Ginebra 1609), que presenta preveer, con el mismo tratamiento:

(3) preueér, preuoir, preuedere, antiuedere, giudicare. (Vittori 1609)

En (4), por su parte se ofrece la entrada para preveer que aparece en el Vocabularium Hispanicum Latinum et Anglicum copiossisimum, de John Minsheu (Londres, 1617). Esta obra se destaca porque en ella puede apreciarse por primera vez la alusión a la formación de este verbo, el cual se hace derivar del latino praevidere y no como una formación moderna con el prefijo pre-:

(4) Preveér. L. praevidere. A. to foresee. (Minsheu 1617)

A continuación registra preveer el Vocabolario español e italiano, de Lorenzo Franciosini (Roma, 1620):

(5) preueer. [ antiuedere. vedere innanzi (Franciosini 1620)

La siguiente obra pertinente es el Diccionario muy copioso de la lengua española y alemana, de Nicholas Mez de Braidenbach, editado en Viena en 1670:

(6) Preveer. Vorsehen / zuvor sehen. (Mez de Braidenbach 1670)

El siguiente diccionario en orden cronológico es el A new Spanish and English Dictionary, de John Stevens (Londres, 1706). Al igual que ocurre en el Vocabularium de Minsheu, Stevens destaca también el origen latino de preveer:

(7) Preveér, to foresee. Lat. Praevidére. (Stevens 1706)

Finalmente, tenemos el Diccionario castellano y portuguez para facilitar a los curiosos la noticia de la lengua latina, de Raphael Bluteau (Lisboa, 1721). En este diccionario nuevamente encontramos como entrada la palabra preveer. Es interesante notar que el término que aparece en cursiva a su derecha (prever) no es una referencia interna a esta entrada en el diccionario, 
como pudiera pensarse, ya que tal entrada no existe, sino que se trata de la traducción ofrecida al portugués. En efecto, este diccionario sigue tal patrón en otras voces como prevención: "Prevencion. Prevençaõ". Esto es sumamente interesante, pues muestra claramente que el autor del texto distingue entre los vocablos preveer y prever y asocia solamente el primero con la lengua española.

(8) Preveer. Prever. (Bluteau 1721)

La primera aparición de la forma prever en el NTLLE data de 1726-1739 y corresponde al Diccionario de autoridades, el primer diccionario de la lengua española creado por la Real Academia Española. A diferencia de lo observado en las ocho obras anteriores, en este texto la entrada corresponde a la voz prever y no preveer, y esta última no aparece mencionada, como puede apreciarse en (9).

(9) PREVER. v.a. Ver con anticipación, conocer por algunas señales ò indicios lo que ha de suceder. Es del Latino Praevidere. EsQuil. Rim. Tercet. À la Passión. De quien el vano errór fuere previsto, / Queriendo ser mayor en el Colegio, / Será la infamia de vender á Christo. (RAE 1726-1739)

La publicación del Diccionario de autoridades marca un claro punto de inflexión en la historia de estas palabras, pues a partir de la fecha de su aparición la variante preveer desaparece completamente de todas las obras contenidas en el NTLLE. Prever es, así, la única voz presente en todos los diccionarios editados por la RAE (tanto en sus versiones manual como usual) entre 1780 y la actualidad, obras que además conservan también con variaciones mínimas la definición entregada en 1726-1739, es decir: "ver con anticipación, conocer por algunas señales o indicios lo que ha de suceder". La influencia de este diccionario (y de sus sucesivas versiones) es patente en las restantes obras lexicográficas presentes en el NTLLE editadas a partir de esta fecha, todas las cuales incluyen la palabra prever, excluyen la variante preveer $\mathrm{y}$, con solo dos excepciones (el diccionario de Terreros y Pando (1767) y el diccionario de Domínguez (1846)), reproducen fielmente la misma definición propuesta en el Diccionario de autoridades. Ofrecemos a continuación las definiciones propuestas en los diccionarios de Terreros y Pando (1767, (10)), Núñez de Taboada (1825 (11)), Salvá (1846 (12)), Domínguez (1846 (13)), Gaspar y Roig (1855 (14)), Zerolo (1895 (15)), Toro y Gómez (1901 (16)), Alemany y Bolufer (1917, (17)), Rodríguez Navas (1918 (18)) y Pagés (1925 (19)).

(10) Prever, ver, ó conocer alguna cosa antes que suceda, conjeturar, adivinar su existencia. (Terreros y Pando 1767) 
(11) PREVER, v. a. Ver con anticipación conocer, conjeturar por algunas señales ó indicios lo que ha de suceder. (Núñez de Taboada 1825)

(12) PREVER. A. Ver con anticipación, conocer, conjeturar por algunas señales ó indicios lo que ha de suceder. Praevidere. (Salvá 1846)

(13) Prever, v. a. Antever, ó ver con anticipación, conocer, calcular, presumir, conjeturar más o menos atinadamente por algunas señales, circunstancias ó indicios lo que se prepara, lo que amenaza ó ha de suceder, lo que parece venirse encima, ir acercándose, estar para sobrevenir, etc. (Domínguez 1846)

(14) PREVER: v. a. : ver con anticipacion, conocer, conjeturar por algunas señales o indicios lo que ha de suceder. (Gaspar y Roig 1855)

(15) PREVER. [De pre, antes, y ver.] a. Ver con anticipación, conocer, conjeturar por algunas señales o indicios lo que ha de suceder. (Zerolo 1895)

(16) Prever, a. Ver con anticipación, conjeturar lo que ha de suceder. (Toro y Gómez 1901)

(17) PREVER. (del lat. praevidere). v. a. Ver con anticipación; conocer, conjeturar por algunas señales o indicios lo que ha de ocurrir. (Alemany y Bolufer 1917)

(18) Prever. a. Ver con anticipación. \| Conjeturar, conocer por indicios o señales lo que ha de suceder. (Rodríguez Navas 1918)

(19) PREVER (Del lat. previdere): tr. Ver con anticipación; conocer, conjeturar por algunas señales ó indicios lo que ha de suceder. (Pagés 1925)

Creemos que la conservación de la definición propuesta por el Diccionario de autoridades en prácticamente la totalidad de las obras posteriores muestra la clara influencia de la RAE, la que, a nuestro juicio, se extendió también al privilegio de la variante prever por sobre preveer. Un dato interesante que confirma la proscripción sufrida por preveer en las obras de la RAE es la inclusión en el Diccionario de la lengua castellana (RAE 1791) de la palabra prevía (y no preveía), en la definición correspondiente a la voz evitar (20)7. Recordemos que, como hemos citado en la sección 2.2, para Rini (1991) la palabra prevía nunca tuvo existencia en el español. A mayor abundamiento, el Corpus diacrónico del español ofrece en su base de datos 
131 apariciones de preveía y solo 3 de prevía, dos de ellas posteriores a 1791 y posiblemente atribuibles, entonces, al influjo académico.

(20) EVITAR. v. a. Precaver que suceda alguna cosa y también librarse alguno con prudencia y previsión del daño, ó perjuicio que le amenazaba, ó de cualquier lance, ruido, ú ocasión en que previa peligro. (RAE 1791)

\subsection{OBRAS LEXICOGRÁFICAS INCLUIDAS EN MEMORIA CHILENA}

Con los propósito de, por una parte, poder acceder a obras que hubieran tratado de las voces prever y preveer fuera del ámbito peninsular, así como también para poder observar su análisis en textos diferentes a diccionarios, hicimos una búsqueda en el sitio web Memoria chilena (http://www. memoriachilena.cl). Este es un centro de recursos digitales que contiene las colecciones de la Biblioteca Nacional de Chile y otras instituciones pertenecientes a la Dirección de Bibliotecas, Archivos y Museos de Chile (DIBAM). Este centro digital ofrece una sección denominada Lexicografía chilena, que contiene los textos de sus archivos dedicados al estudio del léxico, desde 1842 hasta 1940. Consultamos todas las obras disponibles en el catálogo (22 en total) para encontrar en ellas la aparición de las variantes prever o preveer y su tratamiento. Esta pesquisa resultó interesante, pues los libros revisados, como anticipábamos, no corresponden solamente a diccionarios, sino que también se hallan en gran número obras normativas, como lo evidencian títulos como Diccionario manual de locuciones viciosas y de correcciones del lenguaje, de Ortúzar (1893) o Zizaña del lenguaje: vocabulario de disparates, extranjerismos, barbarismos y demás corruptelas, pedanterías y desatinos introducidas en la lengua castellana, de Orellana (1889).

Las obras pertinentes resultaron ser cuatro. Como esperábamos, pues los libros son todos posteriores al Diccionario de autoridades, estos textos consideran como forma única o correcta la voz prever. El primero de ellos, en orden cronológico, es Correcciones lexigráficas sobre la lengua castellana en Chile, de Gormaz (1860), quien incluye en su libro una sección denominada Diccionario ortográfico, consistente en un listado de palabras correctamente escritas, a su juicio. En este listado aparece la palabra prever como una de aquellas que debe escribirse con $v$, pero no consigna preveer.

La siguiente obra pertinente es el Diccionario de chilenismos, de Rodríguez (1875). Este autor señala como voz correcta prever y censura el uso de preveer, como se aprecia en (21): 
(21) PREVER. Los verbos compuestos de ver se conjugan como él. Hai por lo tanto una $e$ demas en los siguientes pasajes: «La ciencia observa las relaciones de causa y efecto que existen entre los hechos i se esfuerza por deducir de esta observación fórmulas que le permitan preveer los fenómenos futuros». (Diego Barros Arana.- Traducción de artículo de M. Courcelle, publicado en La Revista del Pacífico.) ......"puede preveer las consecuencias de tal i tal acto o preveer los hechos venideros.» (ID. ID.) (Rodríguez 1875)

Esta entrada es particularmente interesante por dos motivos: se trata, en primer lugar, de un caso de censura explícita de la forma preveer, $\mathrm{y}$, en segundo término, muestra que esta variante era usada por un hablante culto (Diego Barros Arana, pedagogo, diplomático e historiador chileno) en un texto escrito (traducción de un artículo francés sobre ciencia) en el Chile de finales del siglo xIx, lo que es muestra clara de su vitalidad en la norma culta de ese tiempo.

Preveer aparece censurado en la obra Zizaña del lenguaje (Orellana 1889), como se observa en (22), en que su uso es calificado como "insufrible". Destaca esta entrada porque se resalta en ella el análisis lexicogenésico moderno (pre-ver > prever): "adelantarse por la pre- visión al conocimiento de los hechos futuros", porque en ella se censura el uso de la forma conjugada preveyendo (vigente, al menos hasta el año 1985, como se consigna en La perversión del lenguaje de De Miguel) y porque se ofrece en ella el primer registro que pudimos hallar de la hipótesis de que la presencia de la $e$ "sobrante" proviene de la influencia del verbo proveer. Un último aspecto importante es que Orellana califica la palabra preveer como una "antigualla", lo que deja ver que, a juicio de este autor, se trata de una palabra que sí se escribía o pronunciaba de esta manera en el pasado.

(22) PREVEER.- (Antigualla.) Debe decirse prever, que significa ver antes; adelantarse por la pre-visión al conocimiento de los hechos futuros. Algunos llegan hasta decir preveyendo, lo cual es insufrible. No se confunda la conjugación de este verbo con la de proveer. (Orellana, 1889)

Finalmente, preveer aparece también citado como una voz incorrecta en el Diccionario manual de locuciones viciosas y de correcciones del lenguaje de Ortúzar (1893). Esta entrada, reproducida en (23), destaca por criticar también la inclusión de la forma prevía en el "Diccionario" (suponemos que el autor se refiere a la edición de 1884 del Diccionario de la lengua castellana (RAE), que reproduce la definición de la edición de 1791, observable en (20)), voz a la que califica de "alma en pena", lo que evidencia que se trata de una palabra extremadamente inusual. 
(23) Preveer. Prever. De pre, antes y ver. a. conj. C. ver. Hay por lo tanto una $e$ de más en prevees, prevee, preveí, preveiste; y una de menos en prevía que anda como alma en pena en la voz Evitar del Diccionario. (Ortúzar 1893)

\section{ANÁLISIS DE CORPUS EN EL CORDE}

Complementamos la pesquisa bibliográfica recién presentada con un análisis del Corpus diacrónico del español (CORDE), para datar las apariciones $\mathrm{y}$ frecuencias de uso tanto de prever como de preveer. El período que estudiamos fue desde el año 1000 al 1900, y buscamos en él las ocurrencias correspondientes a las conjugaciones en las tres personas, singulares y plurales, de todos los tiempos verbales del indicativo. Se excluyeron del análisis los participios y formas compuestas, ya que no nos pareció claro si previsto, dada su forma irregular, corresponde al participio de prever o preveer. También descartamos las formas de futuro, ya que estas son menos comunes debido al campo semántico de prever ('ver con anticipación'). Consideramos, además, las variantes ortográficas preuer y preueer, con sus correspondientes conjugaciones, así como formaciones con el prefijo arcaico prae-.

Debe recordarse que este corpus registra solamente usos aparecidos en escritos, los que, por lo general, privilegian las formas más prestigiosas, lo que hace esperable desde el primer momento un mayor número de apariciones de prever, sobre todo en siglos más cercanos al actual.

La aparición más antigua registrada en el CORDE corresponde a un ejemplo de la voz preveer, la que se encuentra en un documento notarial de 1251: "E si nenguna cousa ovier el convento d'essi monesterio, enviar por mí, e soterrarme e preveer mia sepultura". Prever, por su parte, se observa por primera vez casi dos siglos después, en un documento notarial fechado cerca de 1419: "por ende, que ellos, queriendo remediar e prever en los dapnos e males passados e obiar a los que adelante se podían seguir, que ordenavan e ordenaron, de oy día que este estatuto e ordenança es fecha, que nonbrassen dos personas".

A continuación, se presenta una tabla que reúne las frecuencias completas de aparición de ambas formas verbales prever y preveer en el infinitivo, gerundio, presente, pretérito perfecto y pretérito imperfecto desde el siglo XIII al XIX. Desde el siglo xv en adelante, la tabla considera períodos de cincuenta años, para una visión más clara de la evolución del uso de estas palabras. Las frecuencias se ofrecen luego en un gráfico para su mejor apreciación. 


\begin{tabular}{|l|r|r|r|r|r|r|r|r|r|r|r|r|}
\hline & XIII & XIV & $1-X V$ & 2-XV & 1-XVI & 2-XVI & 1-XVII & 2-XVII & 1-XVIII & 2-XVIII & 1-XIX & 2-XIX \\
\hline preveer & 1 & 2 & 2 & 2 & 3 & 3 & 5 & 8 & 12 & 28 & 50 & 81 \\
\hline prever & 0 & 0 & 2 & 1 & 3 & 0 & 14 & 15 & 24 & 63 & 156 & 333 \\
\hline
\end{tabular}

Tabla 1: Usos de prever frente a preveer entre los siglos XIII y XIX

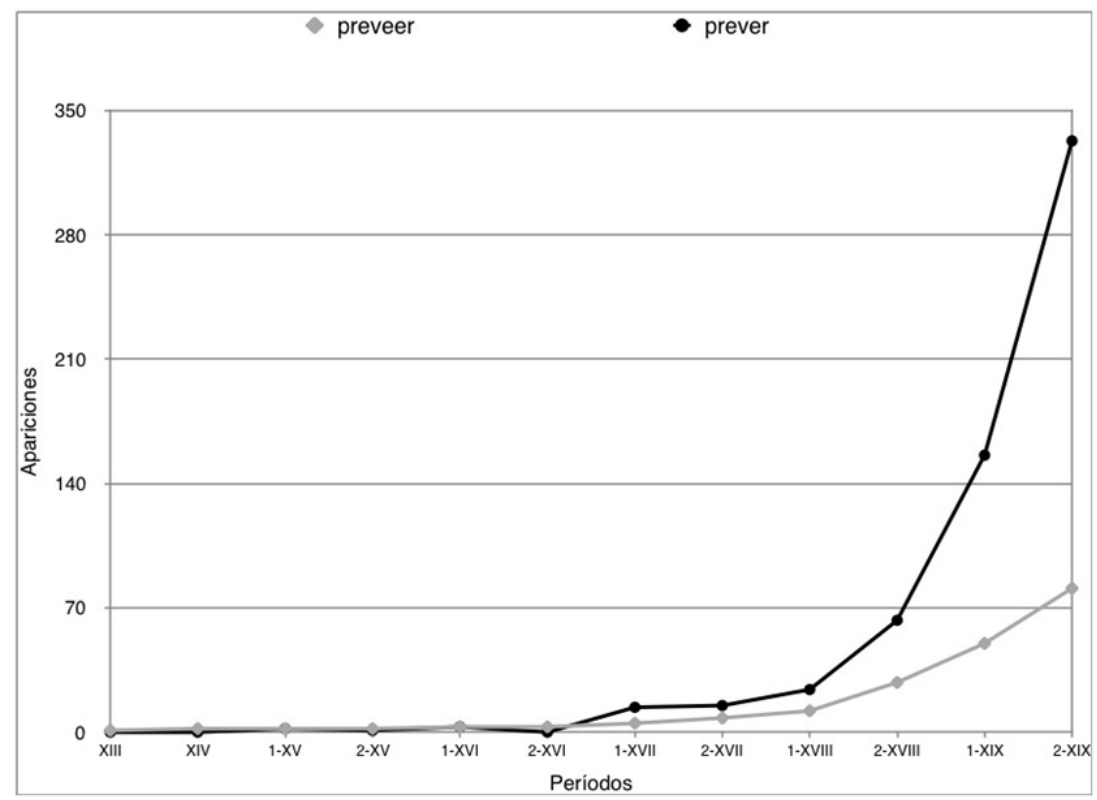

Gráfico 1: Usos de prever frente a preveer entre los siglos XIII y XIX

Podemos observar en este gráfico que ambas formas, si bien manifiestan baja frecuencia de aparición, están en competencia hasta mediados del siglo XVIII. A partir de ese momento, la forma con una sola vocal, vale decir prever, se eleva posicionándose con la mayor frecuencia de uso, diferencia que se hace mucho mayor desde el siglo XIX en adelante. Estos resultados son consistentes con nuestra hipótesis, ya que muestran que el privilegio de prever y el desuso de preveer (al menos en la variante escrita del español registrada en el CORDE) coinciden con la publicación del Diccionario de autoridades en 1726-1739. 


\section{CONCLUSIONES}

Al principio de este artículo planteamos dos hipótesis que podrían explicar la existencia de la forma preveer como variante de prever en el español actual. La primera de ellas es la que se encuentra en varios textos de gramática normativa y dice que preveer se forma por un cruce moderno con la palabra proveer. La segunda, postulada por nosotros, señala que tanto prever como preveer han sido formas existentes en el español, que se mantuvieron en competencia y que preveer solamente vio disminuido su uso (al menos en el español culto) por el influjo de la Real Academia Española, que escoge prever en 1726-1739 en el Diccionario de autoridades y en obras sucesivas. La revisión de las obras lexicográficas mostró que, efectivamente, la voz preveer aparece registrada en un conjunto de ocho diccionarios previos a 1726 como la forma privilegiada y que solo después de esa fecha los diccionarios recogen la variante prever, con definiciones muy semejantes -cuando no idénticas-a las dadas por la RAE en esa fecha. Esto es, a nuestro juicio, un claro argumento a favor de nuestra hipótesis. La revisión de otras obras históricas de carácter normativo sirven para sustentar aún más este punto y para mostrar que la voz preveer (como aparece en el Diccionario de chilenismos de Rodríguez) era usada en documentos escritos por hablantes de elevada cultura hasta bien entrado el siglo XIX. La revisión del Corpus diacrónico del español, si bien con escaso número de datos, muestra también una competencia cercana entre ambas variantes al menos desde el siglo XIII, la que solo se decide en favor de prever en el español culto desde mediados del siglo XVIII en adelante, lo que coincide también con lo planteado en nuestra hipótesis.

Lo dicho hasta aquí es pertinente especialmente para la variedad culta del español. Nos atrevemos a aventurar, sin embargo, que en el habla coloquial la competencia entre prever y preveer no se ha resuelto, sino que ambas variantes se mantienen en pugna hasta hoy. Esto sería refrendado justamente por la frecuente censura que sufre el verbo preveer en obras normativas. Esta hipótesis debería ser confirmada con un estudio sincrónico sobre el uso de ambas variantes.

\section{REFERENCIAS BIBLIOGRÁFICAS}

Academia Chilena de la Lengua. 2007. Notas idiomáticas 35. Santiago: MareNostrum. 2014. Lo pienso bien y lo digo mal. Santiago: Catalonia.

Alcoba Rueda, Santiago. 2012. Morfología de las palabras archisílabas actuales. Quaderns de Filología 77: 15-82. 
Alemany y Bolufer, José. 1917. Diccionario de la lengua española. Barcelona: Ramón Sopena.

Arteta, Aurelio. 1995. La moda del archisílabo. El País [en línea]. Disponible en http:// elpais.com/diario/1995/09/21/opinion/811634406_850215.html [Consulta 15/04/2016]. 2005. Arrecian los archisílabos. El País [en línea]. Disponible en http://elpais.com/ diario/2005/08/10/opinion/1123624805_850215.html [Consulta 15/04/2016].

2008. Archisílabos. El País [en línea]. Disponible en http://elpais.com/ diario/2008/12/16/opinion/1229382004_850215.html [Consulta 15/04/2016].

2010. 'Archisílabos' a tutiplén. El País [en línea]. Disponible en http://elpais.com/ diario/2010/02/05/opinion/1265324404_850215.html [Consulta 15/04/2016].

Bluteau, Rafael. 1721. Diccionario castellano y portuguez para facilitar a los curiosos la noticia de la lengua latina, con el uso del vocabulario portuguez y latino. Lisboa: Pascoal da Silva.

Centro Virtual Cervantes (s. f.). “Prever” es 'ver con antelación' [en línea]. Disponible en http://cvc.cervantes.es/lengua/alhabla/museo_horrores/museo_065.htm [Consulta 23/04/2016].

Corominas, Joan. 1954-1957. Diccionario crítico etimológico de la lengua castellana. Madrid: Gredos.

Corominas, Joan y José A. Pascual. 1991-1997. Diccionario crítico etimológico castellano e hispánico. Madrid: Gredos.

Correa, Jaime. 2011. “¿Prever o preveer?”. El español sin misterios. Disponible en http:// espanolsinmisterios.blogspot.cl/2011/07/prever-o-preveer.html [Consulta 23/04/2016].

Domínguez, Ramón. 1846. Diccionario nacional o gran diccionario clásico de la lengua española, el más completo de los publicados hasta el día. Madrid: Establecimiento Léxico-Tipográfico de R. J. Domínguez.

Fundación de Español Urgente (Fundéu). 2011. Prever, no preveer [en línea]. Disponible en http://www.fundeu.es/recomendacion/prever-no-preveer-1048/ [Consulta 11/03/2016].

Franciosini, Lorenzo. 1620. Vocabolario español e italiano. Roma: Iuan Pablo Profilio.

GASPAR, y RoIG. 1855. Diccionario enciclopédico de la lengua española, con todas las vozez, frases, refranes y locuciones usadas en España y las Américas españolas. Madrid: Imprenta y Librería de Gaspar y Roij.

Gormaz, Valentín. 1860. Correcciones lexigráficas sobre la lengua castellana en Chile seguidas de varios apéndices importantes; dispuestas por orden alfabético y dedicadas a la instrucción. Valparaíso: Imprenta del Comercio.

HornKEns, HeInRICH. 1599. Recueil des dictionnaires françoys, espagnols et latins. Bruselas: Rutger Velpius.

Lathrop, Thomas A. 1980. The evolution of Spanish. An introductory historical grammar. Newark: Juan de la Cuesta.

Mez de Braidenbach, Nicholas. 1670. Diccionario muy copioso de la lengua española y alemana hasta agora nunca visto, sacado de diferentes autores con mucho trabajo y diligencia. Viena: Juan Diego Kürner.

Miguel, Amando DE. 1985. La perversión del lenguaje. Madrid: Espasa.

Minsheu, John. 1599. A dictionary in Spanish and English. Londres: John Haviland.

1617. Vocabularium Hispanicum Latinum et Anglicum copiossisimum, cum nonnullis vocum millibus locupletatum, ac cum Linguae Hispanica Etymologijs. Londres: Joanum Browne.

Nieto Jiménez, Lidio, y Manuel Alvar Ezquerra. 2007. Nuevo tesoro lexicográfico del español. Madrid: Arco Libros. 
NúÑez de Taboada, Melchior. 1825. Diccionario de la lengua castellana para cuya composición se han consultado los mejores vocabularios de esta lengua, y el de la Real Academia Española últimamente publicado en 1822; aumentado con más de 5000 voces o artículos que no se hallan en ninguno de ellos. París: Librería de Seguin.

Orellana, Francisco. 1889. Zizaña del lenguaje: vocabulario de disparates, extranjerismos, barbarismos y demás corruptelas, pedanterías y desatinos introducidas en la lengua castellana. Curazao: A. Bethencourt é hijos.

Ortúzar Montt, Camilo. 1893. Diccionario manual de locuciones viciosas y de correcciones del lenguaje. Santiago: S. Benigno Canavesa: Impr. Salesiana.

Oudin, CÉsAr. 1607. Tesoro de las dos lenguas francesa y española. París: Chez Marc Orry.

PAGÉs, ANICETO DE. 1925. Gran diccionario de la lengua castellana, autorizado con ejemplos de buenos escritores antiguos y modernos, ordenado con arreglo a la última edición de la Real Academia Española y enriquecido con más de diez mil voces, acepciones, frases y refranes que no constan en ningún otro diccionario. Barcelona: Fomento Comercial del Libro.

Palet, IoAn. 1604. Diccionario muy copioso de la lengua española y francesa en el qual son declaradas todas las palabras castellanas y francesas con sus proprias y naturales significaciones sacadas de muchos y muy excelentes autores antiguos y modernos. París: Matthieu Guillemot.

Real Academia Española (s. f.). Nuevo tesoro lexicográfico de la lengua española [en línea]. Disponible en: http://www.rae.es/recursos/diccionarios/diccionariosanteriores-1726-1992/nuevo-tesoro-lexicografico. [Consulta 23/04/2016]

1726-1739. Diccionario de la lengua castellana, en que se explica el verdadero sentido de las voces, su naturaleza y calidad, con las phrases o modos de hablar, los proverbios o refranes, y otras cosas convenientes al uso de la lengua. [Diccionario de autoridades]. Madrid: Imprenta de Francisco del Hierro.

1791. Diccionario de la lengua castellana. Madrid: Viuda de Joaquín Ibarra.

2005. Diccionario panhispánico de dudas. Madrid: Espasa.

Real Academia Española y Asociación de Academias de la Lengua Española. 2009. Nueva gramática de la lengua española. Madrid: Espasa.

Rodríguez Navas, Manuel. 1918. Diccionario general y técnico hispanoamericano. Madrid: Cultura Hispanoamericana.

Rodríguez, Zorobabel. 1875. Diccionario de chilenismos. Santiago: Imprenta de El Independiente.

Rini, Joel. 1991. The Diffusion of/-eé-/>/-é-/in Ibero-Romance Infinitives: creer, leer, veer, preveer, proveer, seer, poseer. Neuphilologische Mitteilungen: 95-103.

2001. The extraordinary survival of Spanish veia: Another facet of analogy revealed. Hispanic review 69(4): 501-525.

SALVÁ, Vicente. 1846. Nuevo diccionario de la lengua castellana que comprende la última edición íntegra, muy rectificada y mejorada del publicado por la Academia Española y unas veintiséis mil voces, acepciones, frases y locuciones, entre ellas muchas americanas, añadidas. París: Librería de don Vicente Salvá.

Seco, Manuel. 1961. Diccionario de dudas y dificultades de la lengua española. Madrid: Espasa.

Stevens, John. 1706. A new Spanish and English Dictionary. Collected from the Best Spanish Authors Both Ancient and Modern. Containing several thousand words more than any other dictionary; with their etymology. Londres: George Sawbridge.

TERREROS y PANDO EstebAN DE. 1767. Diccionario castellano con las voces de ciencias y artes y sus correspondientes en las tres lenguas francesa, latina é italiana. Madrid: Imprenta de la Viuda de Ibarra, Hijos y Compañía. 
Toro y Gómez, Miguel De. 1901. Nuevo diccionario enciclopédico ilustrado de la lengua castellana. Madrid: Librería Armand Colín - Hernando y Cía.

Vittori, Girolamo. 1609. Tesoro de las tres lengvas francesa, italiana y española. Ginebra: Philippe Albert et Alexandre Pernet.

Zerolo, Elías. 1895. Diccionario enciclopédico de la lengua castellana. París: Garnier Hermanos. 